(C) 1985 IEEE. Personal use of this material is permitted. However, permission to reprint/republish this material

for advertising or promotional purposes or for creating new collective works for resale or redistribution to servers

or lists, or to reuse any copyrighted component of this work in other works must be obtained from the IEEE.

IEEE Transactions on Nuclear Science. Vol NS-32, No. 5. October 1985

\title{
THE SUPERCONDUCTING SEPARATED ORBIT CYCLOTRON TRITRON
}

\author{
U. Trinks, W. Assmann, L. Diatl, G. Hinderer, H.ł. Körner, A. Platzer, B. Rehm \\ K. Rieger, C. Riess, R. Savoy, M. Wandinger, W. Wiedemann \\ Physik-Department der Technischen Universität München und Sektion Physik der Universität \\ München, Germany
}

\section{Introduction}

At the Munich Accelerator Laboratory a booster for the existing MP-tandem - the Tritron - is under construction for acceleration of heavy ions lo specific energies up to $21 \mathrm{MeV} / \mathrm{L}$. The Tritron ${ }^{+}$is a separated orbit cyclotron similar to the $50 C^{1,2}$ but with the magnets and cavities both superconducting. The Tritron fits well into the existing laboratory. It is projected to be a prototype to demonstrate the feasibility of this type of cyclotron, which may be suited to overcome the limits of the conventional cyclotron concept. First, there are no axial focusing problems. Secondly, there is no crossing of resonances in the betatron frequency diagram, and thirdly, there are no injection and extraction problems. Thus continuous ion beams of high intensity and high quality with energies up to about $1 \mathrm{GeV} / \mathrm{u}$ seem within reach by connecting several separated orbit cyclotrons with increasing radii in series.

\section{The Tritron Concept}

The Tritron is a superconducting separated orbit cycloLron with injection radius $r_{2}=70 \mathrm{~cm}$ and extraction radius $r_{2}=149 \mathrm{~cm}$, so the energy gain factor is $\sim 4.5$ (ma$x$ imum energy for protons: $\sim 43 \mathrm{MeV}$, for ions with $Q / \mathrm{A}=$ $0.5: \sim 21 \mathrm{MeV} / \mathrm{u})$. The bending system consists of $12 \mathrm{flat}$ sector magnets of $20^{\circ}$ azimuthal width each (see Fig. 1). Each sector is made up of 20 neighbouring window-frame magnets bent along the spiral orbit $(B<1.5 \mathrm{~T})$. It is produced by milling 20 almost concentric slots with a distance of $4 \mathrm{~cm}$ in two sheets of iron and putting them together face by face, after having wound the superconducting high current density coils inside.

In order to achieve the turn separation of $\Delta r=4 \mathrm{~cm}$ the maximum voltage of the accelerating system has to be $U\left(r_{1}\right) \approx 1.4$ MV respectively $U\left(r_{2}\right) \approx 3$ MV per revolution. This will be provided by six superconducting cavities in every second intermediate sector. The wedge shaped cavities with inward directed accelerating gaps - radially extended for simultaneous acceleration of ali 20 beams - are of the reentrant type, driven in the TM010mode.

The magnets and cavities (total weight $\leq 4 \mathrm{tn}$ ) hang on a torus shaped 270 l helium reservoir inside a large vacuum vessel $(\emptyset 3.6 \mathrm{~m})$. When the lower half of the vessel is removed immediate access is given to all parts of the machine. Thus complicated cryostats as well as vacuum chambers for the beam are avoided. Some data of the Tritron are summarized in Tab. 1.

In the Tritron the magnetic field has to fulfil the condition of isochronism as in a conventional cyclotron. But in contrast to cyclotrons the radial gradients of the field in the individual channels are independent from each other and may be chosen according to the requirements of focusing. Thus one gets transversal and longitudinal focusing as in synchrotrans. Note two consequences: First, because there is no need of an dzimuthal field variation for axial focusing the ratio of the maximum value of $B(\theta)$ to the mean field can be made quite small. This effect becomes evident especially at high energies. Secondly, due to the longitudinal focu-

In the Tritron the ions are made to follow a spiral orbit through narrow channel magnets - like the thread, which once was passed by Daidalos through the turns of a TRITON-snail by means of an ant. sing the RF-frequency can be made a rather inign harmonic of the revolution frequency of the ions. Thus the frequency range of the cavities for acceleration of

Table 1: Tritron Data

Injector

Max. energy: $H^{1+}(Q / A=1)$

$\left.S^{16+(0 / A}=0.5\right)$

$33+(Q / A=0.26)$

Energy gain factor

injection radius

Extraction radius

Turn separation

Number of turns

12 magnet sectors with sector angle

Bending field

Superconducting cable

supercond. cable, 14 strands, each. $\emptyset$

Maximum current in the cable $\mathrm{Cu} / \mathrm{NbT} \mathrm{i}$

6 superconducting cavities

RF-frequency

Harmonic number $t$.

Maximum voltage per cavity

Dissipated heat at $4.5 \mathrm{~K}$ per cavity

13 MV-tandem

42.8 iver

$21.3 \mathrm{MeV} / \mathrm{u}$

$5.8 \mathrm{MeV} / \mathrm{L}$

4.5

$70 \mathrm{~cm}$

$149 \mathrm{~cm}$

$4 \mathrm{~cm}$

20

$<1.5 \mathrm{~T}$

$0.7 \times 3.0 \mathrm{~mm}^{2}$

$0.4 \mathrm{~mm}$

1.35

$1320 \mathrm{~A}$

TM010-mode

$170 \mathrm{MHz} \pm 3 \%$

2 19

$500 \mathrm{kV}$

$<6$ W
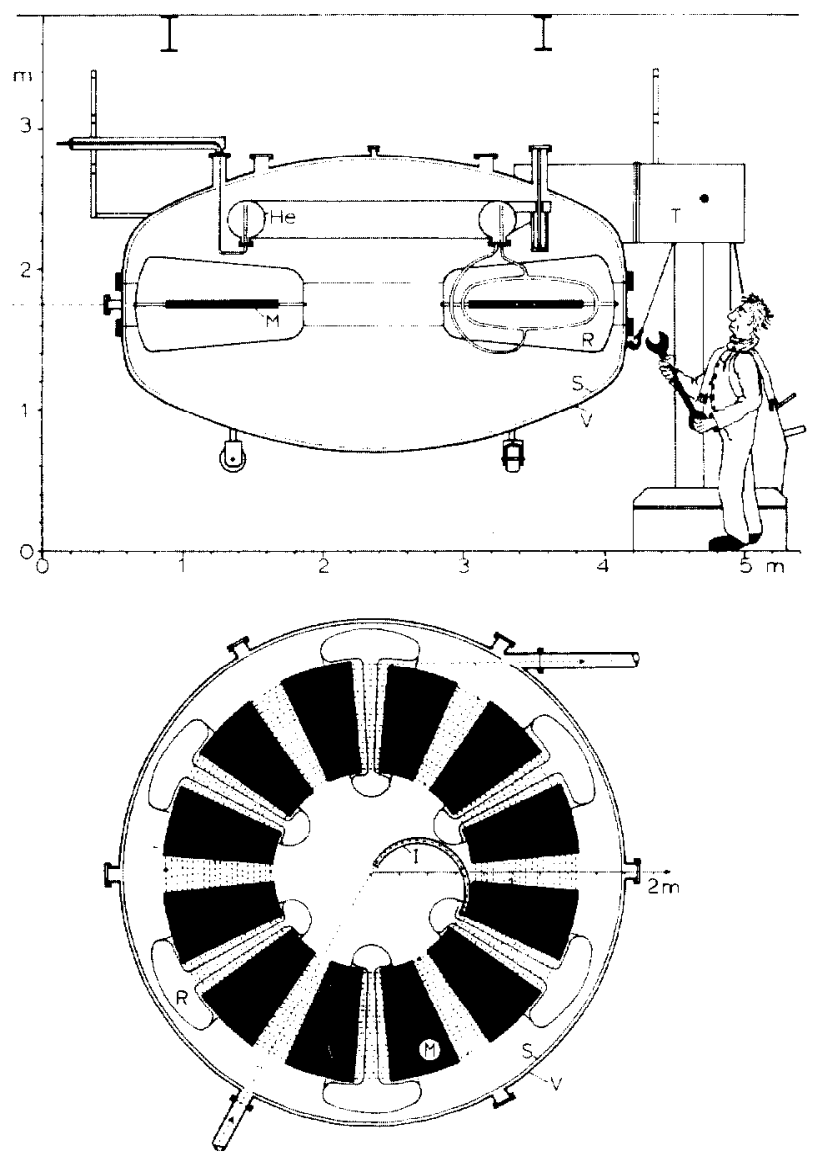

Fig. 1: Cross section of the Tritron. M: magnets, R: RF-acceleration cavities, I: injection magnet, $V$ : vacuum vessel, 5 : $80 \mathrm{~K}$-shield, He: support and liquid helium reservoir. Dotted: ion beam 


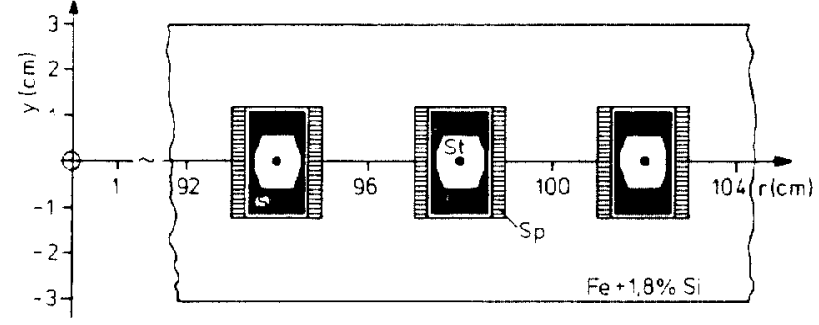

Fig. 2: Radial cross section of a magnet sector. Sp: Superconducting coil, st: beam, S: rad. shield

various beams to different energies will be sufficiently small, so that it call be achieved by simply pressing the cavities in azimuthal direction, thus changing the gap distance and the capacity respectively without sliding contacts.

\section{The Magnets}

As the tandem supplies the Tritron with beams of excellent quality, an aperture of the channel magnets of $\sim 12 \mathrm{~mm}$ suffices. The bending field will be less than $3 \lesssim 1.5 \mathrm{~T}$. Then the permeability of the $1.8 \% \mathrm{Si}$ steel at $4.5 \mathrm{~K}$ is $\mu>3000^{3}$, and the ampere-turn of the coils is less than $1200 \mathrm{~A}$ per mm gap height. A width of the coil of $3 \mathrm{~mm}$ yields an overall current density of $400 \mathrm{~A} / \mathrm{mm}^{2}$, which is easily attainable with superconducting coils because of the low field. Then a total width of the channel magnets of $4 \mathrm{~cm}$ results (see Fig. 2). The coils are potted and cooled via the steel. They are shielded from beam losses by a copper tube inside the channel. The coils consist of 24 turns of a cable $\left(0.7 \times 3.0 \mathrm{~mm}^{2}\right)$ with 14 strands $(\varnothing=0.4 \mathrm{~mm}, \mathrm{Cu} / \mathrm{NbTi}=$ 1.35 ), insulated with two lavers of prepreg. The end of a coil (bedstead type) is shown in Fig. 3 during a test winding (the cable still without insulation). For $B=1.5$ T the cable current is $1320 \mathrm{~A}$, which is about $30 \%$ of the critical current at $4.3 \mathrm{~K}$.

The field gradient in the channels will be constant, the axial focusing is achieved by edge focusing (tilt $\left.9^{\circ}\right)$. The field is homogenous within $\sim 10^{-4}$, if the height of the window is constant and the current distribution is really uniform. If there are gaps of $0.1 \mathrm{~mm}$ between the uppermost and lowest conductor of the coil and the steel frame, the field increases from the center towards the radial edge of the window by $\sim 10^{-3}$. This sextupole term can be used to compensate for the corresponding term of the stray fields at the entrance and exit of the channels. Field calculations (POISSON) show that $3 \%$ change of field in one channel causes $\triangle B / B<10^{-4}$ in the adjacent ones at $1.5 \mathrm{~T}$.

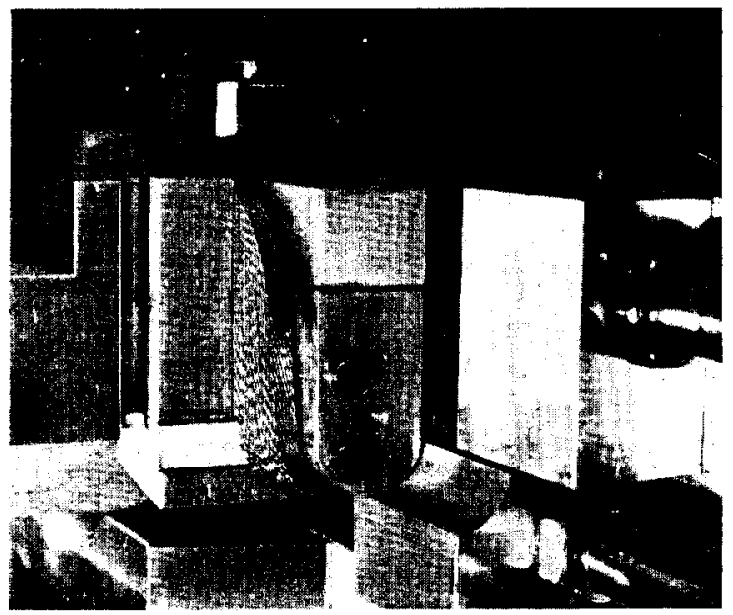

Fig. 3: End of a coil (bedstead type) during a test winding with some of the pneumatic pistons.

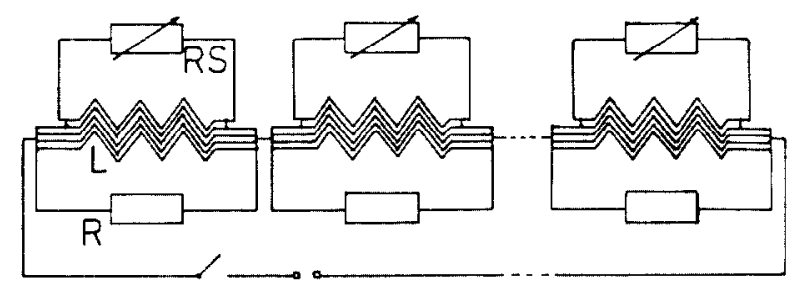

Fig. 4: Scheme of the 236 magnets with $L=0.13$ to 0.25 $\mathrm{mH}$, dump resistors $\mathrm{R}=0.25$ to $0.5 \mathrm{~m} \Omega$ and supercond. Switches with RS $=0$ or $0.05 \Omega$ connected to one of the strands of the cable

The individual currents of all 236 magnets differ from the mean by less than $2 \%$. For all magnets only one power supply is needed, if all coils are connected in series, each with its own cold dump resistor and a superconducting switch in parallel (see Fig. 4). Each switch is put between the ends of one of the strands of the cable with superconducting joints, thus forming a loop with excellent coupling to the coil. If the current $I$ in one coil has to be changed by $\Delta \mathrm{I}$, the current supply is set to $I$, then the switch is made normal conducting, and the current supply is set to the new value $I+\Delta I$. The procedure is terminated by switching back to the superconducting state. In all other magnets with the switches superconducting the fields are kept constant because of induced currents in the superconducting loops.

The switch is a superconducting wire with a Cu90 Ni 10 matrix $\left(\emptyset 0.4 \mathrm{~mm}\right.$, length $\left.\sim 2.5 \mathrm{~cm}, I_{\max } \approx 90 \mathrm{~A}\right)$ the ends of which are cooled $(4.3 \mathrm{~K})$. It can be heated above the critical temperature between two small heating windings (see Fig. 5). The switching times in both directions were measured to be $<0.2 \mathrm{sec}$, the heat input in the normal conducting state is $<1 \mathrm{~mW}$ per switch ${ }^{4}$. The superconducting joints are produced by etching bare the filaments of both wires, which shall be joined, then both bundles are twisted, sticked in a copper casing and pressed (see. Fig. 6). For currents below $I_{\max }$ the resistance of these joints was measured to be $<10^{-15} \Omega$.

\section{The Acceleration Cavities}

The large turn separation of the Tritron leads to a considerable reduction of the costs for the magnetic bending system, on the other hand it causes enhanced effort at the accelerating system. The wedge shaped cavities have a length of $120 \mathrm{~cm}$, a maximum height of
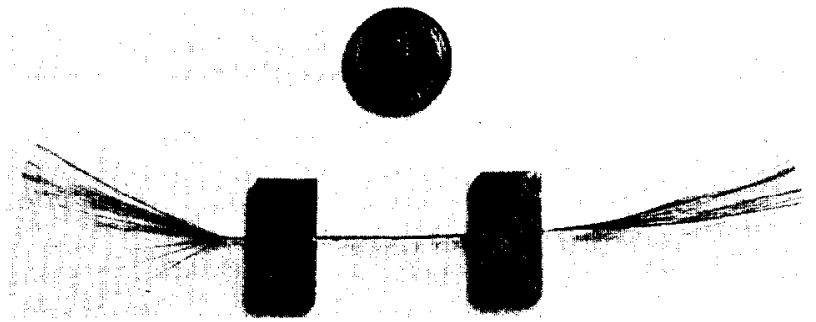

Fig. 5: Superconducting switch with the superconducting filaments at the ends bare of the Cugovi10matrix, soldered on two cooling blocks (Cu) and supplied with heating windings between them.

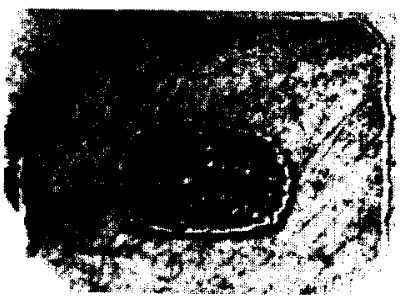

Fig. 6 :

Cross section of a superconducting joint. In the centre the two twisted bundels of NbTi filaments, surrounded by the Cu-jacket (width $\sim 2.5 \mathrm{~mm}$ ) 


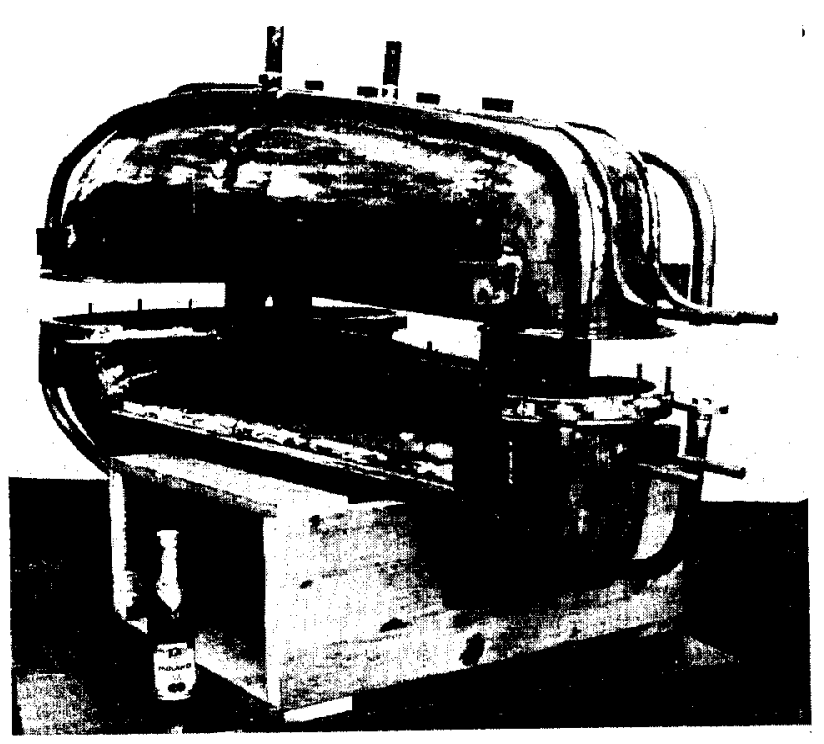

Fig. 7: Tritron cavity under construction.

$70 \mathrm{~cm}$, a gap length of 4 to $8 \mathrm{~cm}$ and a radial gap width of $\sim 85 \mathrm{~cm}$. The frequency is $\sim 170 \mathrm{MHz} \pm 3 \%$ corresponding to harmonic numbers $\geq 19$.

Normal conducting cavities of this type would have a shunt impedance of $54 \mathrm{M} \Omega$, leading to $>375 \mathrm{~kW}$ dissipated heat in the vicinity of the cold magnets. Therefore the cavities are planned to be superconducting. Wi.th a residual surface resistance of $\leq 3 \cdot 10^{-7} \Omega$, which was achieved already with a $500 \mathrm{MHz}$-test cavity ${ }^{5}$, the dissipated heat for all cavities will amount to $\leq 36 \mathrm{~W}$ (total heat input of the Tritron at $4.5 \mathrm{~K}:-40 \mathrm{~W})$. Note that the surface resistance strongly depends on the magnetic background field. Thus the short range of the stray fields of the magnets is essential for the use of superconducting cavities.

The cavities consist of an upper and lower half. They are made of $6 \mathrm{~mm}$ thick OFHC-copper (see Fig. 7), which will be electropolated with a lead alloy as superconductor. For frecuences $<200 \mathrm{MHz}$ the surface resistance is dominated by the residual losses, which depend on the surface preparation but not on the temperature. Thus cooling by boiling helium is sufficient.

From several reasons lead alloy on copper was chosen instead of niobium. First, on account of the large dimensions and complicated shape of the cavities this technique is relatively simple and cheap. Secondly, the thermal conductivity of the thin layer of lead alloy combined with the copper sheets is at least 10 times better than that of massive niobium. Thus indirect cooling is possible with two helium pipes soldered on the cavity (bath cryostats are avoided). In addition the good thermal conductivity helps considerably to hinder the spreading of normal conducting spots on the superconducting surface. Thirdly, lead alloys as PbSn or PbBi are more stable against oxidation than pure lead. Finally, the shape of the Tritron cavities can be optimized with respect to the maximum magnetic and electrical fields, so that the superior critical data of niobium become irrelevant.

The development of superconducting cavities at Munich was started with a test cavity of rotational symmetry $(044 \mathrm{~cm}, v=490 \mathrm{MHz}$ ). The best results until now were achieved with a PbSn-layer. The total surface resistance at $4.5 \mathrm{~K}$ was $5.3 \cdot 10-7 \mathrm{R}$ at a voltage of $600 \mathrm{kV}$ across the gap of $10 \mathrm{~cm}$ length. If a RBCS-resistance of $2.6 \cdot 10^{-7} \Omega$ is assumed, the residual resistance would be $2.7 \cdot 10^{-7} \Omega$. The quality factor was $3.5 \cdot 10^{8}$. More detai Is are reported in another contribution to this conference 5 .

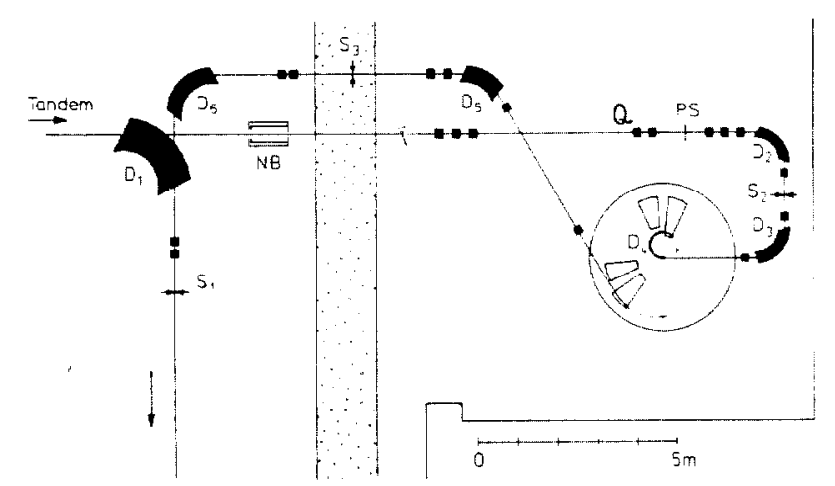

Fig. 8: The beam transfer lines with D: dipol magnets, $Q$ : quadrupol lenses, NB: post buncher, PS: post stripper, S: slits.

\section{Beam Dynamics}

Because of the small aperture of the channel magnets the ion beam has to be well centered throughout the whole machine. The constant turn separation of $\Delta r=4 \mathrm{~cm}$ requires a well defined energy gain per turn depending on the radius. It can be achieved by choosing the proper central (synchroneous) phase with respect to the RF. This leads to a certain phase curve $\phi_{s}(r)$, which is obtained by small shifts of the phase from turn to turn, realised by a corresponding shift of the revolution frequency. This is produced by small $\left(<10^{-3}\right)$ deviations from the isochroneous fields for the central particle. In order to get longitudinal focusing, $\phi_{s}$ has to stay between $90^{\circ}$ and $180^{\circ}$. Computer calculations for a sinoidal dependence of the accelerating voltage $U_{0}(r)$ with $U_{0}\left(r_{2}\right)=U_{\max }$ have shown a typical phase shift from $\sim 140^{\circ}$ at $r_{1}$ to $\sim 110^{\circ}$ at $r_{2}$.

Due to the longitudinal focusing non central particles oscillate with a synchrotron frequency of about 0.1 to 0.5 per turn (thus in total $\sim 5$ to 8 oscillations) in the longitudinal and radial phase space. For the expected longitudinal phase space of the injected beams the corresponding radial width is less than $\sim \pm 3 \mathrm{~mm}$.

The radial and axial betatron frequencies are in the range of 0.8 per turn. The betatron motions are superposed to the synchrotron oscillations, which leads to a total beam width of less than $\pm 4 \mathrm{~mm}$. More details about beam dynamics and tolerance problems are given in 6$)$. In Fig. 8 the beam transfer from the tandem and to the experiments is shown.

The project is partly funded. It is supported by the Federal Government (BMFT).

\section{References}

[1] F.M. Russell, A Fixed-Frequency, Fixed-Field, HighEnergy Accelerator. Nucl. Instr. Meth., 23 (1963) 229

[2] J.A. Martin, The Separated Ortit Cyclotron. IEEE-NS-13 (1966) 288

[3] A. McInturff, J. Claus, Low Temperature Iron Measurements. 3rd int. Conf. Magn. Techn., Hamburg 1970, p. 45

[4] A. Platzer, Entwicklung eines supraleitenden Schalters für die Tritron Magnete Diplomarbeit TUM, May 1985

[5] L. Dietl, K. Rieger, U. Trinks, Lead Alloys for Superconducting Cavities, this conference

[6] G. Hinderer, U. Trinks, Das Tritron. Internal Report, TUM, Dec. 1983 